\title{
Double Closed-loop Control System Design of PMSM Based on DSP MoupengTao ${ }^{1, \text { a }}$,SongjianGuo ${ }^{2, b}$, SongQiang ${ }^{3, c}$ \\ ${ }^{1}$ College of Electronic Information and Control Engineering, Beijing University of Technology, Beijing, 100124, China \\ ${ }^{2}$ College of Electronic Information and Control Engineering, Beijing University of Technology, Beijing, 100124, China \\ ${ }^{3}$ College of Electronic Information and Control Engineering, Beijing University of Technology, Beijing, 100124, China \\ a email: 13167537519@163.com, ${ }^{b}$ email:sjglcy@163.com ${ }^{c}$ email:1239440665@qq.com
}

Keywords: PMSM; Vector control theory; Double closed loop control system;SVPWM;DSP

\begin{abstract}
In this paper, the vector control theory of PMSM is studied, and the double closed-loop control system of PMSM is designed. By analyzing the mathematical model of PMSM, vector control principle and control strategy based on vector control, the control strategy and realization method of this paper are determined. The operating principle and algorithm of space vector pulse width modulation (SVPWM) are analyzed. The SVPWM and the whole control model are simulated by Matlab/Simulink. Finally, the hardware system is built with TMS320F28335 TI as the control core. The control system is tested. The test results prove the practicability and effectiveness of the control system.
\end{abstract}

\section{Introduction}

Vector control system [1] of PMSM is a kind of high performance AC control system, which has the advantages of simple structure, small size, light weight, high efficiency, high overload capacity, small inertia and small torque ripple. Because of the current loop, the current loop is decoupled into DC control, which can achieve DC control performance. The current loop can handle the problem of its pulse. Because of the high speed loop, the current loop is given. The three-phase AC motor is controlled by the vector control theory. The three-phase stator current is decoupled into the torque current and exciting current by the coordinate transformation, and the control strategy is easy to achieve a good control performance[2].

\section{PMSM vector control principle and mathematical model}

Vector control theory solve the problem of AC motor drive strategy including PMSM, so that the AC motor can be controlled like a DC motor. The method is: by means of coordinate transformation, the electric potential of the armature in the stationary two-phase coordinate system can be decomposed into the direct axis electric potential and the quadrature axis. And the two components are mutually perpendicular, so we can adjust them separately in order to achieve the control effect of similar direct current motor[3].

The coordinate transformation is the basis of analyzing the mathematical model of PMSM and its control strategy. Because the coordinate transformation can be used for the AC motor in the three-phase $\mathrm{AC}$ winding is equivalent to two phase vertical $\mathrm{AC}$ winding and two phase rotating $\mathrm{DC}$ winding, which will greatly simplify the analysis and control of AC motor. Vector control in the coordinate system used in three: 


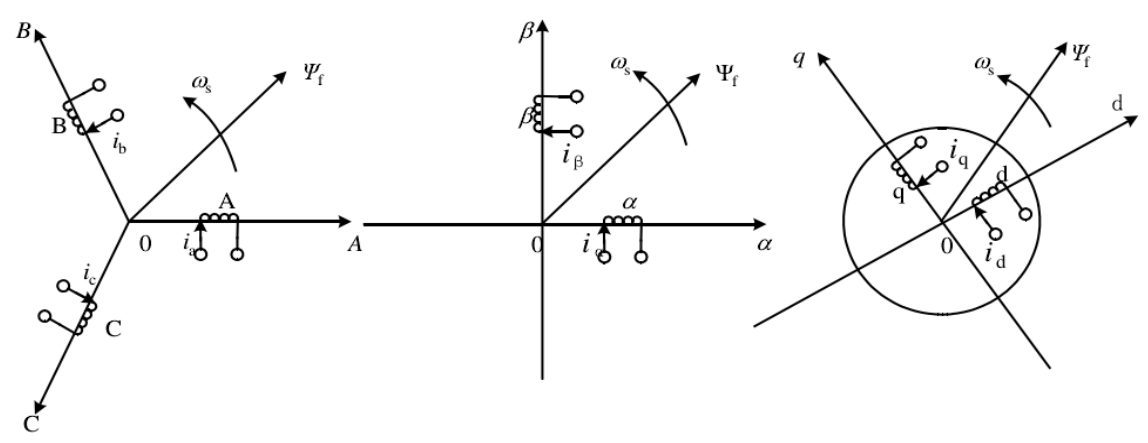

(a)Three phase AC winding (b)Two phase AC winding(c)Rotary DC winding

Fig.1. Physical model of the motor winding

In vector control, the coordinate transformation is mainly refers to the transformation of the three phase static $\mathrm{ABC}$ coordinate system to the two-phase static coordinate system, the Clark transform and the two-phase rotating coordinate system, the Park transform and the inverse transform. By means of coordinate transformation, we can get the mathematical model of the PMSM under the dq coordinate system with the mathematical model of the three phase stationary ABC coordinate system[4][5].

Voltage equation:

$u_{d}=\frac{d \psi_{d}}{d_{t}}-\omega \psi_{q}+R_{s} i_{d}$

$u_{q}=\frac{d \psi_{q}}{d_{t}}+\omega \psi_{d}+R_{s} i_{q}$

Flux linkage equation:

$\psi_{d}=L_{d} i_{d}+\psi_{f}$

$\psi_{q}=L_{q} i_{q}$

Electromagnetic torque equation:

$T_{e m}=\frac{3}{2} p\left(\psi_{d} i_{q}-\psi_{q} i_{d}\right)=\frac{3}{2} p\left[\psi_{f} i_{q}+\left(L_{d}-L_{q}\right) i_{d} i_{q}\right]$

Motor motion equation:

$T_{e m}-T_{L}=\frac{J}{p} \frac{d \omega}{d t}$

The electromagnetic torque equation is divided into two parts. The first part is the electromagnetic torque generated by the interaction between the current and the permanent magnet. In the second part, it is caused by the effect of the rotor, which is called the reluctance torque. Under the effect of rotating magnetic field of stator, the asymmetry of the magnetic field of the cross shaft and the axis of the shaft is proportional to the difference between the shaft and the shaft. So we can control the size and direction of the electromagnetic torque by controlling the size and direction of the DQ axis current, and then control the motor rotation.

\section{Double closed-loop control system design and SVPWM}

The PMSM control system is composed of current loop and speed loop. And the performance of the whole system is the basis of the whole system performance, and the performance of the outer ring is dependent on the optimization of the inner loop. Current loop is the basic of high performance control system. The dynamic response of the system is directly related to the 
realization of vector control strategy, and also directly affects the dynamic performance of the whole system. Speed loop is also an extremely important link. The control performance is an important part of the system control performance[6]. In a broad sense, speed servo control should have high accuracy and fast response characteristics.

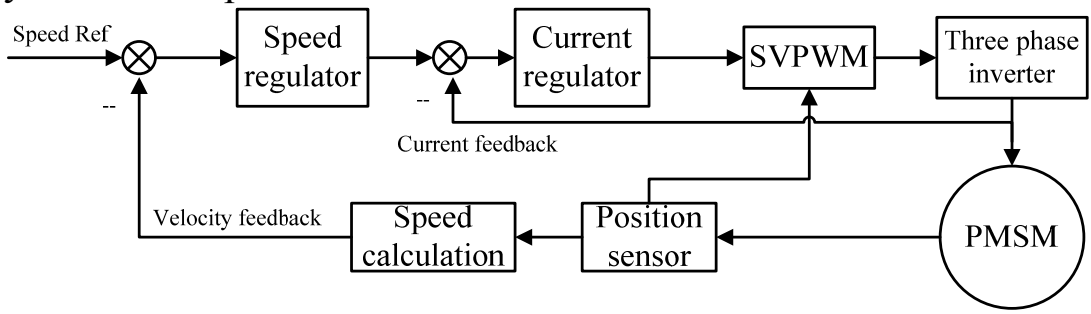

Fig.2. Double closed loop control system structure diagram

According to the dynamic structure diagram of the motor, the speed loop is opened. The electromagnetic time constant of the motor is much less than the mechanical time constant. And because the anti electromotive force and speed is proportional to the speed loop, but a slow change in the current loop in the process of rapid adjustment, it can be considered. This does not take into account the effect of the potential feedback, thus simplifying the current loop structure, and the transfer function of the current loop of the controlled object is: $G_{s}=\frac{1 / R s}{T_{s}+1}$ After the current regulator is added, the dynamic structure of the current loop is shown in Fig3[7].

After the current regulator is corrected, the transfer function is a typical I type. The current loop and the controlled object are used as the control object in the speed loop. After correction of the open loop function: $G_{s}=K_{N} \frac{\tau_{n} s+1}{s^{2}\left(T_{\Sigma_{n}} s+1\right)}$ After correction, the dynamic structure of the speed loop is shown in Fig4.

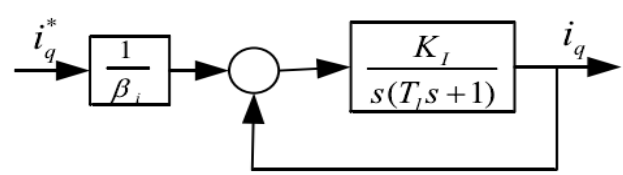

Fig.3.Current loop dynamic structure

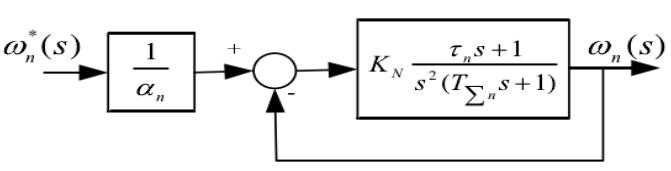

Fig.4.Dynamic structure diagram of speed loop In summary, according to the specific parameters of permanent magnet synchronous motor, the speed current controller can be designed according to the engineering setting method, and the speed current controller is designed, and then the PI parameters are determined according to the actual situation. Double closed loop system topology is shown in Fig5:

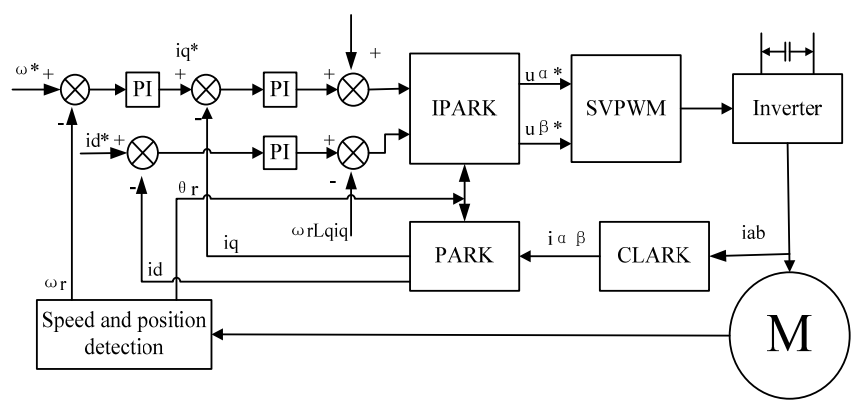

Fig.5.Double closed loop system topology

Vector Pulse Width Modulate Space (SVPWM) is an important part of vector control. Through frequency conversion to achieve the motor speed control strategy, the motor control of the regulator is adjusted, the final will be transformed into PWM as the inverter switch device on the conduction and off signal. As the last part of the control strategy, the performance of the control system is very important. SVPWM has the advantages of high utilization rate, low harmonic components and low power consumption. It can be used in combination with the vector control algorithm to maximize the performance of the device. Therefore, the application of SVPWM in motor frequency conversion speed control system is more frequent[8]. 


\section{System simulation}

After the research and analysis of the permanent magnet synchronous motor and its control strategy, we need to simulate the whole control system, and set provide reference for our design. we establish the simulation model of the coordinate transformation module, SVPWM module and control system in Matlab/Simulink environment. Finally, the whole control system is simulated and the simulation results are analyzed.

First, the PMSM plus a fixed load torque, at the same time given an electromagnetic torque value, the simulation results are shown in the following figure:

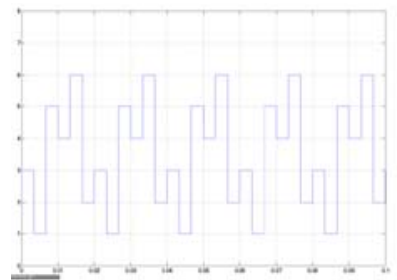

(a)Sector $\mathrm{N}$

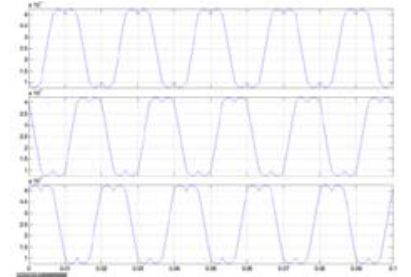

(b) Time switching point

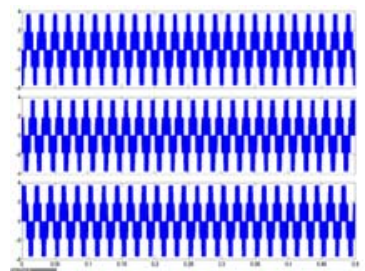

(c) Phase voltage Fig.6.Simulationg results of SVPWM

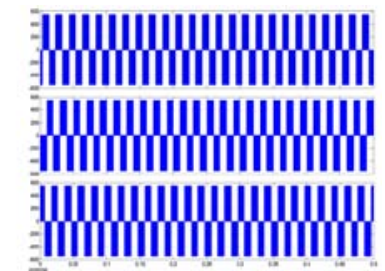

(d)Line voltage

From the above picture (b), we can see that the modulation waveform obtained by SVPWM modulation is saddle shaped, which is beneficial to improve the voltage utilization . From the (d) line voltage waveform we can get, the SVPWM modulation mode can make the line voltage peak is equal to the DC bus voltage, relative to the SPWM, improve the voltage utilization. SVPWM can better control the inverter, so that the phase voltage is 6 step wave.

PMSM plus a fixed load torque, while a given electromagnetic torque values, the simulation results obtained as shown below:

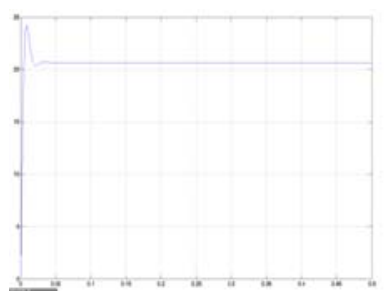

(a)rotational speed

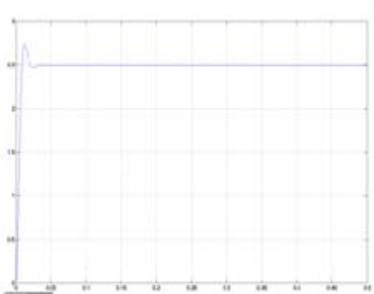

(b) torque

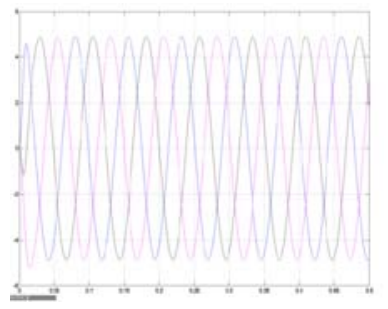

(c) phase current

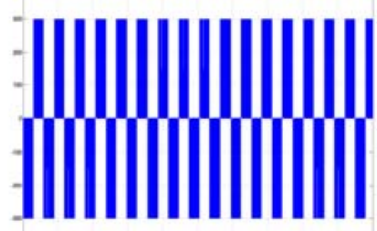

(d) line voltage

Fig.7.Simulationg results of constant torque

From the above (a) and (b) can be seen, the motor speed of the motor increases with the torque, the final drive torque and load torque to achieve a balance, speed is also stable at a fixed value. (c) in the phase current of the simulation shows that the amplitude of the phase current increases, the final magnitude of the same, and the frequency is not the same as the line voltage waveform, amplitude also reached the DC bus voltage value, the frequency remained unchanged.

Secondly, given the PMSM load torque, the torque is given, the simulation results are shown in the following diagram:

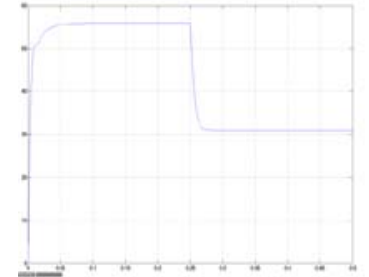

(a)rotational speed

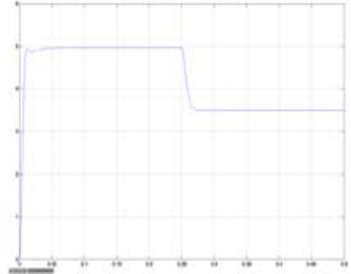

(b) torque

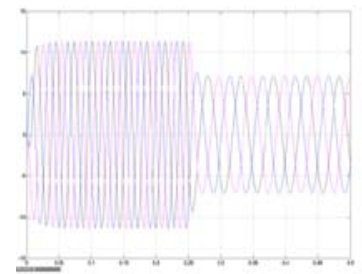

(c) phase current

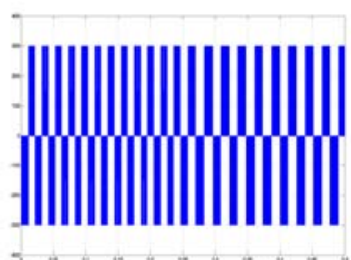

(d) line voltage

Fig.8.Simulationg results of variable torque

Due to the beginning of the torque is greater than the load torque, speed up, and then stabilized at a fixed value, when the simulation time reaches $0.25 \mathrm{~s}$, the torque value of the given value is small, the driving torque and the load torque is not balanced, the speed and then decreased, and finally stable at the other fixed speed, drive torque and load torque to achieve balance. We can see from the 
three phase current waveform, the given value of the torque is reduced, the stator current amplitude is reduced, and the frequency is also reduced. The width of the line voltage waveform is also increasing, and the frequency is decreasing.

\section{Hardware design and Test results}

The whole control experiment platform has the following parts: the host computer, the main control board, the isolation drive board, the IPM inverter module and the PMSM. The main control board and IPM module between the need to add a separate driver board to isolate the driver signal. The main control board is mainly on the sampling circuit, communication circuit, level conversion circuit, rotating transformer decoding circuit and the main control chip TMS320F28335 minimum system circuit. The main circuit of the system is supplied by DC power supply, and the IPM inverter module is supplied to the PMSM power supply, and the driving signal of the inverter is provided by the main control board which is separated by the six PWM signal. The rotor position of the PMSM has a rotating transformer to detect, and the detection signal passes through the SPI bus and the main control chip is in communication with the main control chip. The host computer through CAN communication to transmit system given signal and control switch signal, and receive a variety of sampling signal.

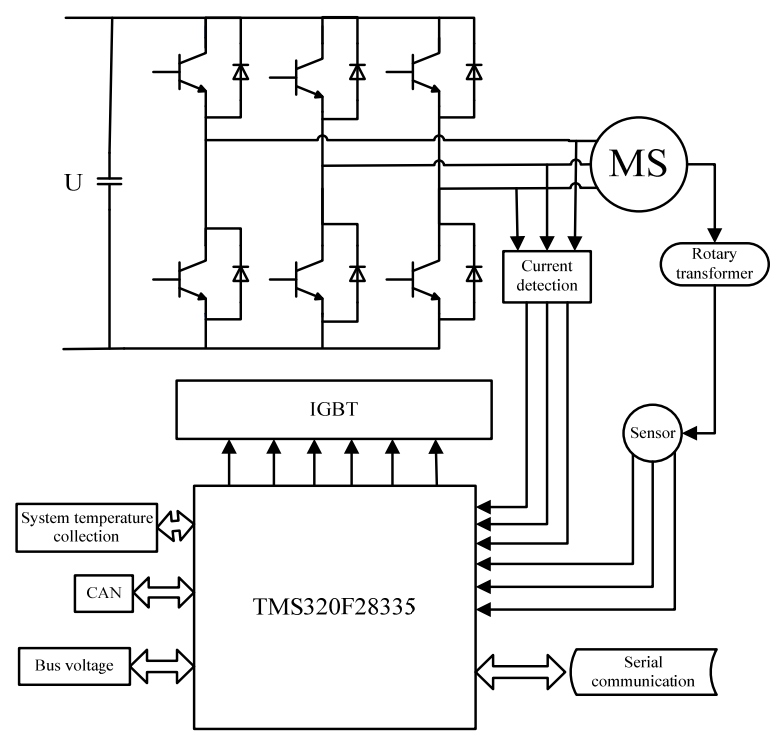

Fig.9.Hardware system diagram

The motor control program is downloaded to the DSP, and the CCS compiler is debugged online, and the output of the sub modules is observed. CCS not only can monitor the changes of the variables, but also provides a graphical monitoring interface, which can observe the change of a variable. The DSP output waveform received IGBT driver, according to the principle of the IGBT main circuit design: if you want to open the IGBT security and reliability, the driving voltage should be $+15 \mathrm{~V}$ and $-8 \mathrm{~V}$, as shown in figure. DSP output of the PWM signal for $+3.3 \mathrm{~V}$ and $0 \mathrm{~V}$, after driving the circuit can output $+15 \mathrm{~V}$ and $-8 \mathrm{~V}$ drive signal, indicating that the driver circuit design to achieve the desired requirements.

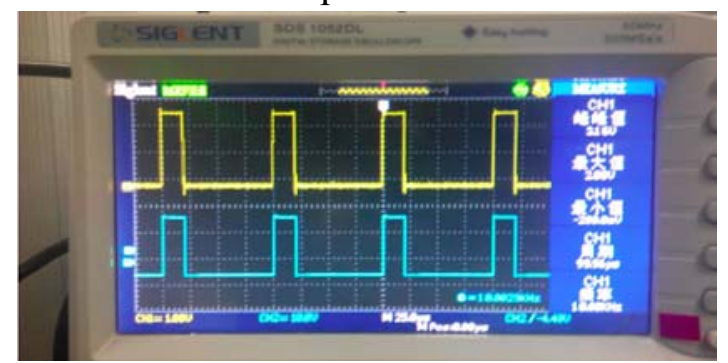

Fig.10.Driver board output SVPWM waveform

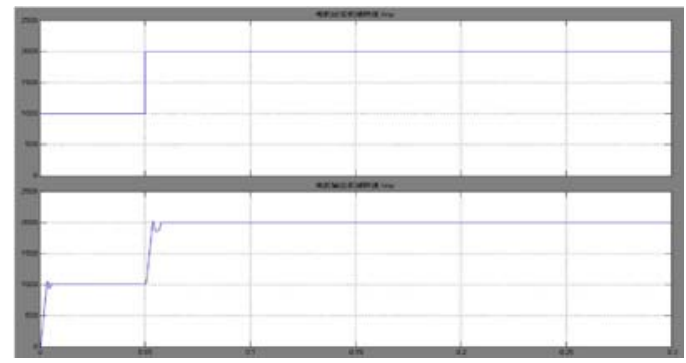

Fig.11.Speed closed loop 


\section{Conclusion}

In this paper, the principle of vector control is introduced. Based on the mathematical model of PMSM in rotating dq axis coordinate system, the vector control principle and vector control scheme are described in detail. Based on the control scheme of this paper, the hardware system based on TMS320F28335 is built, and the control system is tested. The experimental results show that the control system is feasible and correct.

\section{References}

[1] Wang Chengyuan. Vector control AC servo drive motor [M]. Machinery Industry Press, 1995

[2] Morimoto S, Takeda Y, Hirasa T, et al. Expansion of operating limits for permanent magnet motor by current vector control considering inverter capacity[J]. Industry Applications, IEEE Transactions on, 1990, 26(5): 866-871.

[3] Tang RenYuan Modem permanent magnet machines theory and design)[J]. 1997.

[4] Pillay P, Krishnan R. Modeling of permanent magnet motor drives[C]//Robotics and IECON'87 Conferences. International Society for Optics and Photonics, 1987: 289-293.

[5]Morimoto S, Takeda Y, Hirasa T, et al. Expansion of operating limits for permanent magnet motor by current vector control considering inverter capacity[J]. Industry Applications, IEEE Transactions on, 1990, 26(5): 866-871.

[6] LiJian .Design and implementation of the three closed loop AC permanent magnet synchronous motor servo system based on DSP [M]University of Electronic Science and technology.2007

[7]Jagadish H Pujar, S.F. Kodad,Digital Simulation of Direct Torque Fuzzy Control of PMSM Servo System[C],International Journal of Recent Trends in Engineering,2009:89-93

[8]Zhang Qinglu.Design of control system for permanent magnet synchronous motor[M]Beijing University of Technology,2013 